\title{
Simulation of Mechanics Technology Training Course Based on VIRTOOLS
}

\author{
Yang Yang ${ }^{1}$, Bo Yang ${ }^{2}$, Yiqiong $\mathrm{He}^{1}$ and Bin Shen ${ }^{3, *}$ \\ ${ }^{1}$ The PLA rocket force command college, China, 430010 \\ ${ }^{2}$ Information Communication Acdemy of National University of Defense Technology, China, 430212 \\ ${ }^{3}$ Wuhan Donghu University, China, 430212 \\ ${ }^{*}$ Corresponding author
}

\begin{abstract}
Mechanics technology training course, it can comprehensively use mechanics technology and other related theories and practical knowledge of completed courses. It can carry out systematic design and training for students. It can further consolidate, deepen and expand these knowledge. In practical training, there are many limitations in training courses, such as site assignment, course time arrangement, training equipment update, etc. It can not provide necessary help for college students to fully adapt to social needs. This paper studies how to apply virtual reality technology to Mechanics training course. The virtual training system has great significance for the training course of mechanics equipment. It is an important guarantee for the training of qualified personnel. It effectively solves many problems in training, such as complex structure, high cost, and the limitation of field, quantity and type, and low training efficiency.
\end{abstract}

Keywords—simulation; mechanics; Virtools; practical training

\section{INTRODUCTION}

Virtual reality technology is based on virtual reality technology. It takes computers and their corresponding hardware devices as experimental means. It can realize the training process of mechanics. It uses computer simulation and virtual reality technology. It establishes a "solid", "field" and "real-time" virtual environment for mechanical equipment training through collaborative working mode. It can effectively provide advanced experimental environment and simulation tools for mechanical and electrical integration equipment operation training. It can be used for drill before the actual operation of the mechanics equipment. Through training in the virtual maintenance laboratory, it can achieve the purpose of enhancing the decision-making and control ability of each stage of the product life cycle and the whole system of the product.

Compared with traditional equipment training, virtual reality training has considerable advantages in many aspects, such as improving training flexibility, breaking through training conditions and reducing equipment maintenance costs. Virtools 5 software is powerful and easy to operate. It is very suitable for the development of similar systems. In particular, in order to improve the training quality, improve the training environment and improve the training environment, we can develop a more realistic and practical virtual maintenance training in order to improve the training quality, improve the training environment, and develop a more realistic and practical virtual maintenance training. Practice system.

On the basis of analyzing and summarizing the design methods of virtual reality technology and traditional Mechanics training course, this paper puts forward the basic framework of virtual reality training. The research plan should be able to give the corresponding design process of virtual reality, and can illustrate the implementation process of the proposed system through case studies ${ }^{[1-8]}$.

\section{Design of the Simulation Maintenance System}

Virtual reality technology is a new technology emerged in recent years. It uses a computer to generate a realistic three-dimensional virtual world, which can bring people a sense of visual, listening and touching, and the user is as if they are in the world, freely observing and manipulating the virtual world and interacting with them naturally. In the virtual world established by VR technology, users are no longer restricted by time and space, and have a "immersive" experience in the sense of touch, hearing and vision. Many universities and scientific research institutions have successfully developed virtual laboratory, roaming system, virtual digital library and digital exhibition hall by using virtual reality technology. In view of the shortcomings of the existing virtual product display system development mode, it is necessary to put forward a kind of convenient, realistic, and relatively simple production method, so that users can understand the comprehensive information of the product intuitively and deeply, and can participate in the new product display mode of product design interactively.

Virtual reality training can provide advanced experimental environment and simulation means for equipment basic introduction and Mechanics design, and it can cultivate the ability of college students to understand the basic features of equipment quickly. Applying virtual reality technology to mechanics equipment, developing virtual reality training system is of great significance for the training of mechanics equipment. It effectively solved the problem of mechanical and electrical integration theory curriculum divorced from practical training, the limitation of field and quantity, and low training efficiency. Therefore, the virtual reality training system is designed to provide effective solutions for the virtual reality training system designed by mechanics system. 
This research will use Visual $\mathrm{C}++$ and Virtools 5 of Dassault company of France as the development platform of the system. Using Dassault company's Solidworks 2012 3D design software and 3dsmax 2015 as modeling and animation generating tools, Access 2010 stores knowledge base, sample part library, special tool library, testing tool library for system database[9-16].

Virtual reality training is the directional simulation under virtual environment, which is closely related to the theme of equipment training. Virtual reality technology includes parts assembly simulation technology, equipment detection simulation and virtual design simulation technology.

The key research of this project is how to realize the virtual reality design of equipment training. Later, it will work mainly around it.

\section{MECHANICAL AND ELECTRICAL INTEGRATION TRAINING DESIGN BASED ON VIRTOOLS}

The system simulates the real operating environment as much as possible. The system chose the training subject of ball wire post clearance adjustment to carry out virtual experiment.

\section{A. Model Construction of Ball Screw Pair}

Virtual training is based on the shape and precision characteristics of the ball screw pair, which simulates the three-dimensional adjustment process of the product, and allows the user to control the three-dimensional real simulation gap adjustment process in an interactive way, in order to test the assembly of the product. The present thought is also an analytical process.

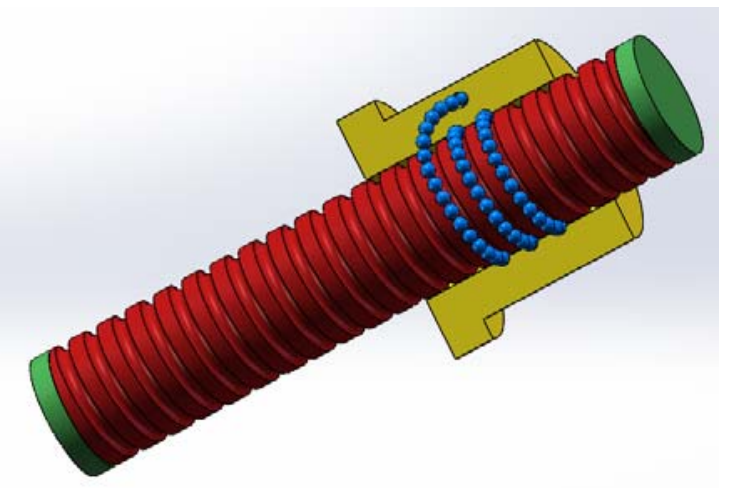

FIGURE I. MODELING OF BALL SCREW PAIR

The 3D model is the foundation of the whole virtual assembly, which directly affects the reality of the virtual environment. Because Virtools has no modeling function, we need to use modeling software to build 3D solid models. But because this article is a mechanical product model, we first use the professional mechanical 3D drawing software AutoCAD2014 for modeling, and then import the built model into the 3dsmax2012, and then from 3dsmax2012 to Virtools 5. Before establishing the model, we should make a preliminary analysis, decompose the complex mechanical products into several simple parts, and then do the three-dimensional modeling of the components. After the model is established and successfully introduced into the 3dsmax2012, the model is given the material and the map, thus increasing the authenticity of the model, reducing the unnecessary polygons and improving the refresh rate of the display.

\section{B. The Establishment of Virtual Experimental Environment}

There is a strict regulation for the commissioning of mechanical equipment. A reasonable arrangement of experimental environment is the key to standardize students' movements. Through three-dimensional layout, we can build a virtual experiment environment rationally. Build virtual operation platform, virtual tool and virtual fixture. So that students can immerse in an approximate real operation environment, carry out Mechatronics experiments, and further enhance the experimental results. In addition. One of the problems that must be considered in virtual experiment is reasonable experiment. We can set up the tracking mechanism of the experiment process to ensure the correctness of students' operation.

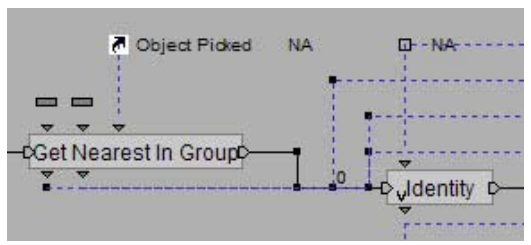

FIGURE II. DISTANCE JUDGEING

The construction of the virtual experiment of the ball screw pair. It is based on the analysis and decomposition of existing experiments. The result of adjustment by equipment. These processes are activated by the output data. In this way, different virtual adjustment operations are performed. Virtual clearance adjustment simulation is the reproduction of actual adjustment process in virtual environment. We must have a reasonable description of clearance adjustment process to guide simulation. The description of the whole process of clearance adjustment should first include the sequence information of the activities of the organization. The relationship between the various installation work, the activity and the detailed and complete human movement and action information and the human-computer interaction information should be expressed in accordance with the timing.

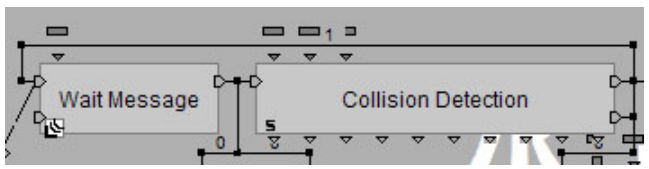

FIGURE III. COLLISION JUDGEING

In the design of interactive virtual gap adjustment, one aspect should be simulated in accordance with the actual installation steps and adjustment methods. On the other hand, the process of real installation should be simplified to realize the purpose of familiarize the students with the adjustment of the clearance and the timing of the adjustment. The implementation of the interactive maintenance process is realized mainly through the call programming to the $\mathrm{BB}$ module: first to create the scripts of the specified object; and then program according to the prescribed action. For some more complex and special operations, you can program by calling the Run VSL module using the Virtools scripting 
language VSL (Virtools Script Languge) or through Virtools's SDK, as shown in Figure 4.

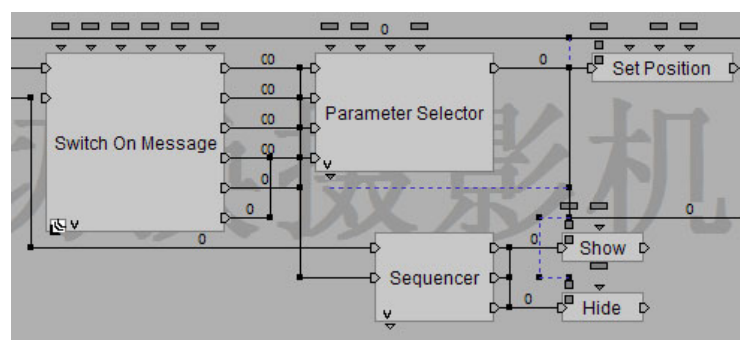

FIGURE IV. TRANSLATION FOR THE CAMARA

In the process of virtual maintenance design, the operation platform, scene and ball screw pair model are introduced into Virtools 5 first, the basic parameters of lighting and camera are set up, and then the program is compiled through the BB module of Virtools, and the virtual simulation of the actual maintenance process is carried out according to the maintenance process, and the better design effect is achieved.

\section{Virtual Judgment of Distance}

The key technology of virtual operation is component collision and assembly distance discrimination. They can be realized by the special collision BBS and design VSL provided by Virtools 5 . The performance of ball screw pair depends largely on the rationality of clearance adjustment. Timing is often guaranteed for beginners to maintain equipment maintenance. Depending on Virtools 5 and $\mathrm{VC}++6$, we design a process to match equipment duty service as VSL, that is training task. The support of the equipment is carried out through virtual reality. If the rubbing oil can be done with different materials and light, the regulation of the control system can be established by setting up virtual instruments, setting regular steps according to the requirements and so on.

\section{Virtual Demonstration and Virtual Display}

Complex equipment through three-dimensional structure, sectional view and so on can well display the structural features of the equipment, connection, and can also master the movement through the way of animation. This will lay a solid foundation for a comprehensive understanding of the structure of the equipment. The demonstration program of virtual assembly and virtual maintenance is established through Virtools 5, and the existing knowledge points are demonstrated. The student's operation rules. The realization of this part is mainly to grasp the transformation of camera lens. Light angle of view and equipment material. As shown in Figure 4.

\section{CONCLUSION}

Compared with traditional experiments, virtual experiments have considerable advantages in improving training flexibility, breaking training conditions and reducing equipment maintenance costs. The software of Virtools 5 is powerful and convenient to operate. It is very suitable for the development of similar system. In particular, it can improve the quality of the students' training, improve the training environment and improve the training environment by using the virtual simulation training to improve the quality of the students' training, and improve the training environment. A more realistic and practical virtual experiment training system is developed step by step.

\section{REFERENCES}

[1] T. M. Cheng, "A fast parametric deformation mechanism for virtual reality applications”[J], Computer \& Industrial Engineering, 2008, 10(10):18-26.

[2] G Antonino, Zachmann Gabriel. Virtual realilv as a tool for verification of assembly and maintenance process[J]. Computer and Graphics, 1999, 23(3):389-403.

[3] Olive Riedel \& Ralf Breining. Engineering Application in Handbook of Virtual Environments Technology[M]. K Stanney, Editor Lawrence Ertbanm Associates, Inc. 2000.

[4] Jeffrey L Wampler, et. Integrating Maintainability and data development[C]. In: Proceedings of the Annual Reliability and Maintainability Symposium, Tamp Bay, 2003.255-262.

[5] Pan Zhigeng, Cheok Adrian David. Virtual Reality and Mixed Reality for Virtual Learning Environments[J]. Computer \& Graphics, 2006, 30(1):20-28.

[6] Daniel Cohen-Or.A survey of visibility for walk through applications[J]. IEEE Transactions on Visualization and Computer Graphics. 2003, 9(3):412-431.

[7] Li J R,Khoo P.Tor B.Desktop Virtual Reality for Maintenance training: An Object Oriented Prototype System(V-REALISM)[J].Computerin Industry, 2003, 52(2): 109-125.

[8] Christopher M C,David C.Virtools Dev User Guide:Version 4.0[z].Virwols SA, 2006.

[9] Virtools 5.0 Online Heferenee.

[10] Richens P,Nitsche M.Mindstage:Towards a ftmetional virtual architecture[C].Computer Aided Architectural Design Futures proceedings,2005:331-340.

[11] Wutthikornthanawat w'Jinuntuya P,Rongviriyapanieh S,et a1.Multi-user tangible interface for public participation development of low-cost housing project design and planning[C].Proeeedings of the 12th International Conference on Computer-Aided Architectural Design Research in Asia-Digitization and Globalizatin,2007:37-43.

[12] Wang Xi,An Yang.Development ofgame software based on Virtools[J].Control and Management,2007,5(5):160-162.

[13] Li Xunxiang,Chen Dingfang,Wang Le,et a1.A development framework for V'trtools-based DVR driving systemiC].Computer Supported Cooperative Work in Design,2007:188-196.

[14] Pan VLin Z,Hu Z,et a1.Research on distributed multi.screendis.play technique based on Virtools[C].7th Intemational Conferenee on Computer-Aided Industrial Design and Conceptual Design, 2006:1-6.

[15] D.U.Silverthom.Teaching and learning in the interactive classroom [J]. Advances in Physiology Education, 2006, 12(30): 135-140.

[16] White, M., Jay, E., Liarokapis, F., Kostakis, C., Lister, P. F. A Virtual Interactive Teaching Environment(VITE)Using XML and Augmented Reality[J]. The International Journal of Electrical Engineering Education, 2001, 38(4): 316-329. 